This item was submitted to Loughborough's Research Repository by the author.

Items in Figshare are protected by copyright, with all rights reserved, unless otherwise indicated.

\title{
Numerical continuation applied to landing gear mechanism analysis
}

PLEASE CITE THE PUBLISHED VERSION

http://dx.doi.org/10.2514/1.C031247

\section{PUBLISHER}

(c) American Institute of Aeronautics and Astronautics

\section{VERSION}

AM (Accepted Manuscript)

\section{PUBLISHER STATEMENT}

This work is made available according to the conditions of the Creative Commons Attribution-NonCommercialNoDerivatives 4.0 International (CC BY-NC-ND 4.0) licence. Full details of this licence are available at: https://creativecommons.org/licenses/by-nc-nd/4.0/

\section{LICENCE}

CC BY-NC-ND 4.0

\section{REPOSITORY RECORD}

Knowles, James A.C., Bernd Krauskopf, and Mark H. Lowenberg. 2015. "Numerical Continuation Applied to Landing Gear Mechanism Analysis". figshare. https://hdl.handle.net/2134/18688. 


\title{
Numerical Continuation Applied to Landing Gear Mechanism Analysis
}

\author{
J. A. C. Knowles ${ }^{* \dagger}$ B. Krauskopf ${ }^{\dagger}$ and M. H. Lowenberg* \\ Faculty of Engineering, University of Bristol, UK
}

\begin{abstract}
A method of investigating quasi-static mechanisms is presented and applied to an overcentre mechanism and to a nose landing gear mechanism. The method uses static equilibrium equations along with equations describing the geometric constraints in the mechanism. In the spirit of bifurcation analysis, solutions to these steady-state equations are then continued numerically in parameters of interest. Results obtained from the bifurcation method agree with the equivalent results obtained from two overcentre mechanism dynamic models (one state-space and one multibody dynamic model), whilst a considerable computation time reduction is demonstrated with the overcentre mechanism. The analysis performed with the nose landing gear model demonstrates the flexibility of the continuation approach, allowing conventional model states to be used as continuation parameters without a need to reformulate the equations within the model. This flexibility, coupled with the computation time reductions, suggests that the bifurcation approach has potential for analysing complex landing gear mechanisms.
\end{abstract}

\section{Introduction}

Mechanisms are structures that move in a pre-determined and controlled manner. They make up an essential part of any aircraft, performing jobs as diverse as deploying aerodynamic surfaces or allowing passengers to adjust their seats. The landing gear mechanism is of particular importance, its purpose being to enable the wheels of an aircraft to move between retracted and deployed states when required to do so by the pilot. To this end, the mechanism needs to downlock to withstand ground loads on touchdown and whilst taxiing. The primary way to deploy and downlock the landing gear is by means of the landing gear actuator, which deploys the gear in a steady and controlled manner. Regulations require a secondary means of deploying the landing gear, to be used should the primary actuator fail to operate. Generally, the simplest and most efficient option is to use gravity as the secondary deployment mechanism: the landing gear simply falls out of the body of the aircraft, and the mechanism downlocks under the freefall motion of the landing gear.

All landing gear mechanism designs must operate reliably in both normal and emergency situations. For a conventional main landing gear with a single side stay, the analysis of its operation under different operational conditions is challenging. With the increasing use of composite materials in aircraft primary structural components, dual sidestay landing gears are being developed to facilitate main landing gear integration within a composite wing box structure. The dual sidestay landing gear is a more complicated mechnism, and presents new challenges to ensure downlocking of both sidestays under any operational conditions, including freefall emergency deployment.

Most of the research into landing gear modelling focuses on dynamic modelling of structural aspects, ${ }^{1,2}$ whereas the analysis of landing gear mechanisms is much less prevalent in the literature. Mathematical analysis of landing gear mechanisms primarily deals with the geometric design of the mechanism, i.e. how to allow the gear to move between two positions (retracted and deployed) within stowed space constraints. ${ }^{3-5}$ The standard approach used by industry to investigate landing gear mechanisms is to perform multiple time history simulations. A slowly varying (quasi-static) force is applied to unlock the lock links, and then re-lock the lock links. The value at which they re-lock is referred to as the downlock force, signified when the

\footnotetext{
* Department of Aerospace Engineering

${ }^{\dagger}$ Department of Engineering Mathematics
} 
angle between the two lock links jumps through $180^{\circ}$. Another parameter of interest, such as the side-stay attachment point, can then be changed before the simulation is repeated. This time history approach uses complex models and requires a large amount of computation time to investigate the system, because a new simulation has to be run every time a parameter value is changed. The choice of parameter that can be varied continuously is also limited; in this instance to the force applied to the lock-links.

An alternative to this 'brute force' computational method is proposed here, which makes use of concepts from the theory of dynamical systems; $\operatorname{see}^{6-8}$ as exemplars for background information. Several recent applications of dynamical systems methods have demonstrated the advantages that they can offer in an aerospace context; this includes the analysis of aircraft ground dynamics ${ }^{9}$ and the study of nose landing gear shimmy. ${ }^{10}$ Here, the mechanism configuration and internal force distribution is formulated as a system of coupled equations, which are inherently nonlinear due to the geometric constraints. The steady-state solutions of these equations can then be found and followed, or continued, in parameters of interest with standard numerical continuation software such as the package AUTO. ${ }^{11}$ A particular advantage of this coupled-equation approach is that any model state (such as an angle between links) or parameter (such as an applied force) can be used as the main continuation parameter without needing to reformulate the governing equations as a function of this main parameter. It is this flexibility, in combination with a substantially lower computational cost, that makes the continuation approach appealing for the analysis of even complicated landing gear mechanisms. The method is first demonstrated in Section II for an overcentre mechanism; this simple example, consisting of a system of 6 equations, is used to compare the dynamic simulation and the numerical continuation approaches. In Section III the continuation method is demonstrated with a nose landing gear model described by a system of 25 equations. This more realistic example demonstrates the versatility of the new method to analyse landing gear downlock loads.

\section{The Overcentre Mechanism}

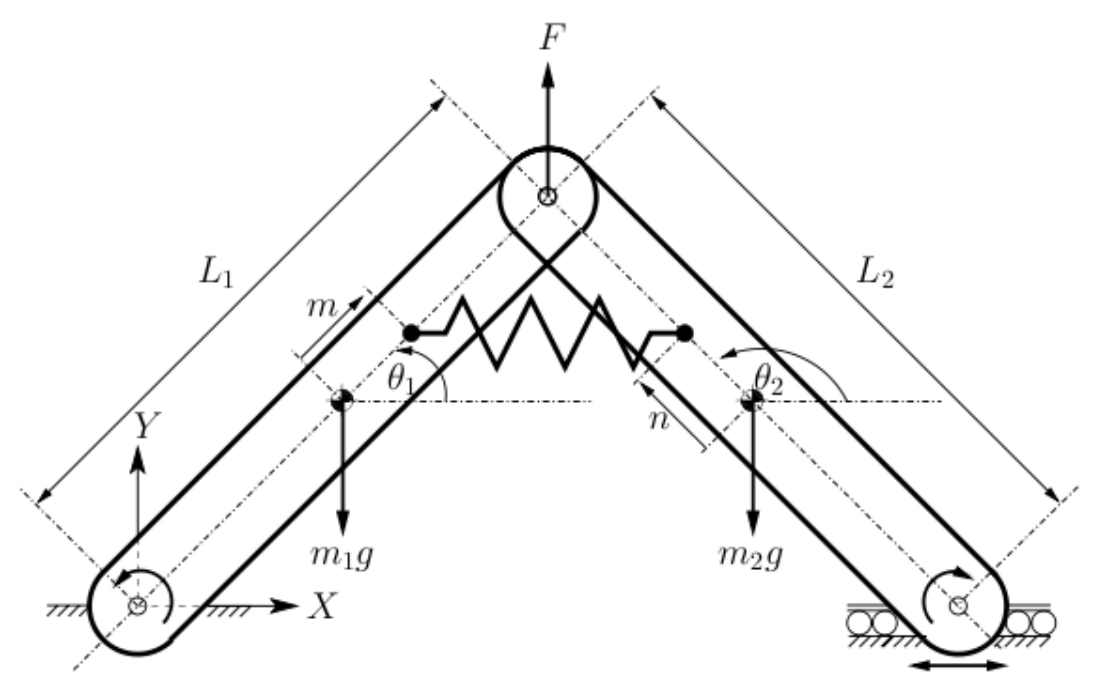

Figure 1. Overcentre mechanism.

The overcentre mechanism as modelled is shown in Figure 1. This planar mechanism consists of two rigid bars attached to each other at one end by a rotational joint. The other end of one of the bars is connected to the ground with a rotational joint - this bar has been designated as bar 1. Bar 2 is also attached to the ground, but via a translational/rotational joint. A spring, attached between the two bars, resists the mechanism movement through the horizontal plane. Three different mathematical models of this overcentre mechanism were created; one state-space dynamic model implemented in Matlab using the inbuilt ODE45 solver, one multibody dynamic model created in ADAMS (a commercial dynamic simulation package) and the new, static-algebraic model created in Matlab. Details of the two Matlab models are presented below. The first Matlab model is a dynamic model with 12 states (bar position, orientation and velocities), formulated using the method of analytical dynamics derived by Udwadia and Kalaba. ${ }^{12}$ The ADAMS model was used to verify the formulation of the dynamic Matlab model. Both models were created without any friction 
to ensure that they would match provided the state-space equations of the Matlab model were formulated correctly. The static algebraic model has 6 states (bar centre of gravity positions and orientations) and is formulated from the geometric constraints and Newtonian force/equilibrium equations. The continuation software package $\mathrm{AUTO}^{11}$ is used to solve this system of algebraic equations by tracing solutions to the steady state equations as a parameter is varied continuously.

\section{A. State-space Dynamic Model}

The state-space dynamic model of an overcentre mechanism was used as the baseline against which to evaluate the static model. The equations of motion were constructed using the fundamental equation as derived by Udwadia and Kalaba; ${ }^{12}$ following their notation:

$$
\ddot{\mathbf{x}}=\mathbf{a}+M^{-\frac{1}{2}}\left(A M^{-\frac{1}{2}}\right)^{+}(b-A \mathbf{a})
$$

Here:

$\mathbf{x}=\left[x_{1}, y_{1}, \theta_{1}, x_{2}, y_{2}, \theta_{2}\right]^{T} ;$

$\mathbf{a}$ is a $(q \times 1)$ external acceleration vector of the unconstrained system (where $q$ is the number of states, which is 6 for the overcentre mechanism);

$b$ is a $(p \times 1)$ vector from the right-hand side of (appropriately differentiated) constraint equations when written in the form $A(x, \dot{x}, t) \ddot{x}=b(x, \dot{x}, t)$ where $p=$ the number of states minus the system degrees of freedom (D.o.F);

$M$ is the $(q \times q)$ mass/inertia matrix of the system;

$I$ is the $(q \times q)$ identity matrix;

$A$ is the $(p \times q)$ matrix from the left-hand side of the constraint equation.

Terms with a superscript ' + ' are the Morse-Penrose (M-P) inverse of that matrix. The Matlab function pinv is used to calculate this inverse in the model; for more information on the M-P inverse see ${ }^{12}$.

The system constraints at the bar ends are specified from the geometry as

$$
\left.\begin{array}{rl}
x_{1}-\frac{L_{1}}{2} \cos \theta_{1} & =0 \\
y_{1}-\frac{L_{1}}{2} \sin \theta_{1} & =0 \\
x_{1}+\frac{L_{1}}{2} \cos \theta_{1}-\left(x_{2}+\frac{L_{2}}{2} \cos \theta_{2}\right) & =0 \\
y_{1}+\frac{L_{1}}{2} \sin \theta_{1}-\left(y_{2}+\frac{L_{2}}{2} \sin \theta_{2}\right) & =0 \\
y_{2}-\frac{L_{2}}{2} \sin \theta_{2} & =0
\end{array}\right\}
$$

Equations (2) are differentiated twice with respect to time before being re-arranged into the form $A(t, x) \ddot{x}=$ $b(t, x, \dot{x})$ to yield

$$
\left[\begin{array}{rrrcrr}
1 & 0 & \frac{L_{1}}{2} \sin \theta_{1} & 0 & 0 & 0 \\
0 & 1 & -\frac{L_{1}}{2} \cos \theta_{1} & 0 & 0 & 0 \\
1 & 0 & -\frac{L_{1}}{2} \sin \theta_{1} & -1 & 0 & \frac{L_{2}}{2} \sin \theta_{2} \\
0 & 1 & \frac{L_{1}}{2} \cos \theta_{1} & 0 & -1 & -\frac{L_{2}}{2} \cos \theta_{2} \\
0 & 0 & 0 & 0 & 1 & -\frac{L_{2}}{2} \cos \theta_{2}
\end{array}\right]\left[\begin{array}{c}
\ddot{x_{1}} \\
\ddot{y_{1}} \\
\ddot{\theta_{1}} \\
\ddot{x_{2}} \\
\ddot{y_{2}} \\
\ddot{\theta_{2}}
\end{array}\right]=\left[\begin{array}{r}
-\frac{L_{1}}{2}{\dot{\theta_{1}}}^{2} \cos \theta_{1} \\
-\frac{L_{1}}{2}{\dot{\theta_{1}}}^{2} \sin \theta_{1} \\
\frac{L_{1}}{2}{\dot{\theta_{1}}}^{2} \cos \theta_{1}-\frac{L_{2}}{2}{\dot{\theta_{2}}}^{2} \cos \theta_{2} \\
\frac{L_{1}}{2}{\dot{\theta_{1}}}^{2} \sin \theta_{1}-\frac{L_{2}}{2}{\dot{\theta_{2}}}^{2} \sin \theta_{2} \\
-\frac{L_{2}}{2}{\dot{\theta_{1}}}^{2} \sin \theta_{2}
\end{array}\right] .
$$

The matrix $A$ and vector $b$ from the left- and right-hand side (respectively) of Eq. (3) can then be used in the fundamental equation (1).

The applied acceleration vector a contains both the gravitational acceleration $g$ and the spring acceleration $a_{s}$. Gravity was assumed to act in the negative $y$-direction with a value of $9.81 \mathrm{~m} / \mathrm{s}^{2}$, whilst the spring acceleration follows the standard differential equation for a linear spring, $M_{r} \ddot{\mathbf{x}}+c \dot{\mathbf{x}}+k=0$. The acceleration caused by the spring is applied between the spring attachment points at distances $m$ and $n$ from the centre of gravity (C.G.) positions of the left- and right-hand bars, respectively; see Figure 1. For simplicity, initial values of 0 for $m$ and $n$ were used, resulting in the applied acceleration vector

$$
\mathbf{a}=\left[\begin{array}{llllll}
a_{s} & -g & 0 & a_{s} & -g & 0
\end{array}\right]^{T} .
$$


The acceleration caused by the spring force is given by

$$
a_{s}=-M_{r}^{-1}\left(k\left(1-\frac{R}{L}\right) H\left[\begin{array}{c}
x_{1} \\
x_{2}
\end{array}\right]+c H\left[\begin{array}{c}
\dot{x_{1}} \\
\dot{x_{2}}
\end{array}\right]\right)
$$

Here:

$M_{r}$ is the reduced mass matrix, containing just the bar masses $\left[\begin{array}{cc}m_{1} & 0 \\ 0 & m_{2}\end{array}\right]$;

$k$ is the spring stiffness;

$R$ is the unstretched spring length;

$L$ is the spring length;

$H$ is the direction matrix used to assign acceleration direction: $\left[\begin{array}{rr}1 & -1 \\ -1 & 1\end{array}\right]$;

$c$ is the damping coefficient.

\section{B. Static Model}

The static model is formulated as a system of simultaneous algebraic equations. The six states are assumed to be independent, with the equations providing the dependencies given by

$$
\left.\begin{array}{rll}
x_{1}-\frac{L_{1}}{2} \cos \theta_{1} & = & 0 \\
y_{1}-\frac{L_{1}}{2} \sin \theta_{1} & = & 0 \\
x_{2}+\frac{L_{2}}{2} \cos \theta_{2}-\left(x_{1}+\frac{L_{1}}{2} \cos \theta_{1}\right) & = & 0 \\
y_{2}+\frac{L_{2}}{2} \sin \theta_{2}-\left(y_{1}+\frac{L_{1}}{2} \sin \theta_{1}\right) & = & 0 \\
y_{2}-\frac{L_{2}}{2} \sin \theta_{2} & = & 0 \\
F_{s x 1}\left[\left(\frac{1}{2}-\frac{m}{L_{1}}\right) \tan \theta_{1}-\left(\frac{1}{2}-\frac{n}{L_{2}}\right) \tan \theta_{2}\right]+F_{s y 1}\left(\frac{m}{L_{1}}-\frac{n}{L_{2}}\right)+\frac{1}{2}\left(w_{1}+w_{2}\right)-F & = & 0
\end{array}\right\}
$$

The first five rows of Eq. (6) provide the inter-state dependencies and would be sufficient to describe the geometry of the system if a single state was specified. They have been derived in the same way as those used to create the dynamic model, given in Eq. (2). The final row results from the static force/moment equilibrium which the mechanism is assumed to maintain in a steady-state. This last constraint enables the inclusion of the overcentre mechanism force $F$, which is initially chosen as the user-varied system parameter.

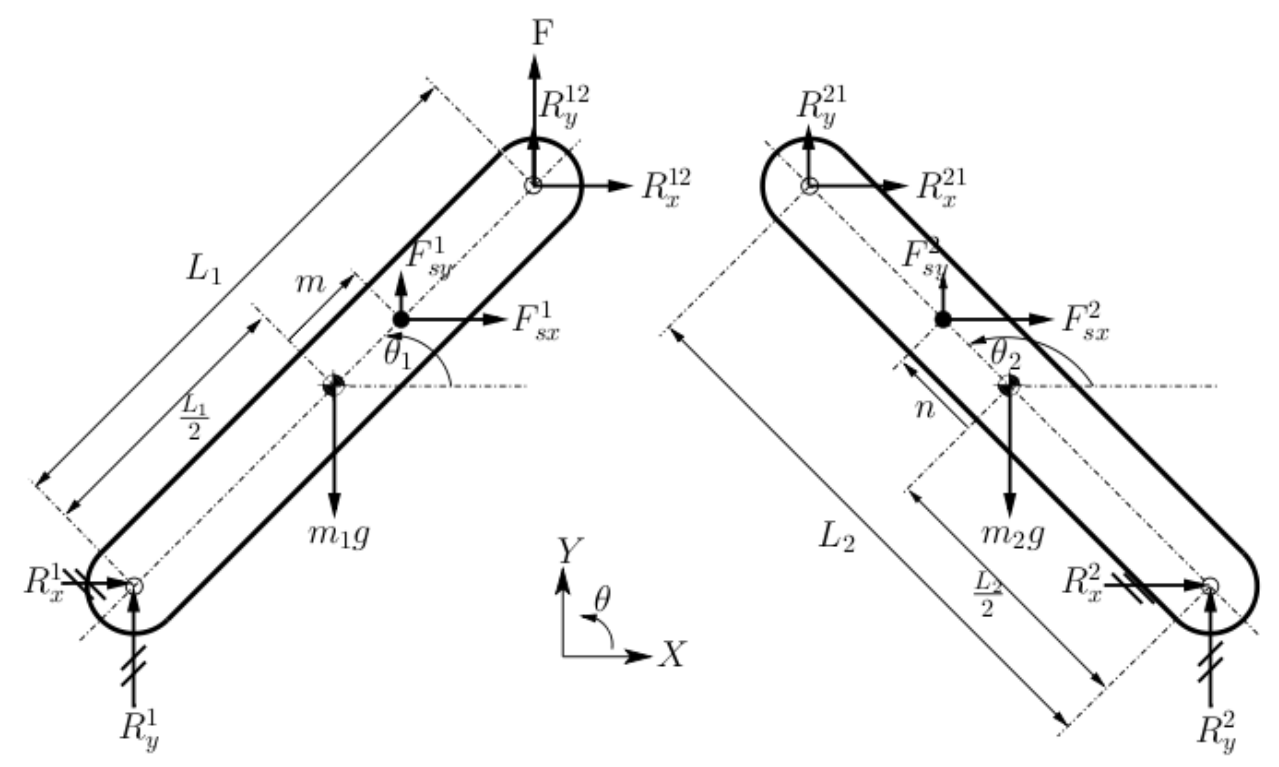

Figure 2. Free-body diagram of overcentre mechanism. 
Figure 2 shows the free-body diagram for the components of the overcentre mechanism which were used to construct the static force/moment equilibrium equations for the static model. The moment equilibrium equations for each bar in terms of the bars' orientations $\theta_{1}$ and $\theta_{2}$, the gravitational forces on the bars $m_{1} g$ and $m_{2} g$ and the spring force components in the global x- and y-directions $F_{s x}$ and $F_{s y}$, are

$$
\left.\begin{array}{rl}
{\left[L_{2} R_{y}^{21}-\frac{L_{2}}{2} m_{2} g+F_{s y}^{2}\left(\frac{L_{2}}{2}+n\right)\right] \cos \theta_{2}-\left[L_{2} R_{x}^{21}+\left(\frac{L_{2}}{2}+n\right) F_{s x}^{2}\right] \sin \theta_{2}} & =0 \\
{\left[L_{1}\left(R_{y}^{12}+F\right)-\frac{L_{1}}{2} m_{1} g+F_{s y}^{1}\left(\frac{L_{1}}{2}+m\right)\right] \cos \theta_{1}-\left[L_{1} R_{x}^{12}+\left(\frac{L_{1}}{2}+m\right) F_{s x}^{1}\right] \sin \theta_{1}} & =0
\end{array}\right\}
$$

Moments were taken about the ground attachment points for each bar to remove the need to include all the reaction forces in the force expression. Due to the nature of the prismatic/rotational joint at the ground point of bar 2 , the horizontal ground force $R_{x}^{2}=0$, so resolving horizontally for bar 2 provides the relation $R_{x}^{21}=-F_{s x}^{2}$.

\section{Comparison of Static and Dynamic Equation Formulation}

Results obtained from the three overcentre models are presented in this section. An investigation is used to demonstrate the efficiency of the new method using the static-algebraic formulation when compared to the traditional approach.

\section{Overcentre Mechanism Model Validation}
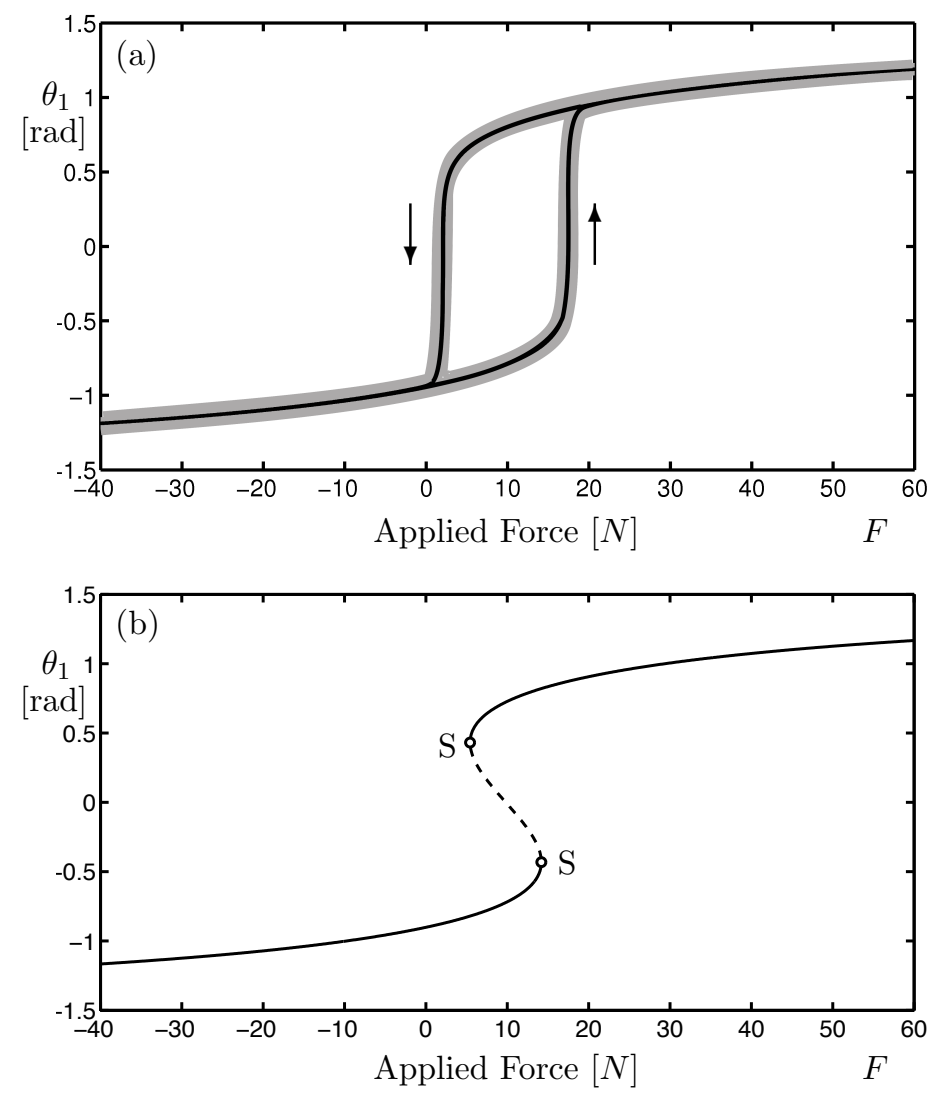

Figure 3. Comparison of quasi-static analysis results from: (a) dynamic models in ADAMS (grey curve) and Matlab (black curve), (b) static algebraic model - stable solutions are indicated by the solid curves.

Figure 3 compares results from three different models. Figure 3(a) shows results from the two dynamic models (in ADAMS and Matlab), where the applied force $(F)$ was gradually decreased and then increased over the displayed range. This figure shows perfect agreement between the two dynamic models over the entire range of applied force $F$. Because of this agreement, the Matlab model was used when comparing 
static and dynamic formulations as it was easier to compare results within the same program without having to export data between programs. Figure 3(b) shows the result of the continuation analysis performed on the static model. The stable steady-state solution branches (solid parts of the curve) match the dynamic results well; the unstable steady-state solution branch cannot be found directly with dynamic simulations.

The result from the static model can be used to explain the nonlinear behaviour observed in the dynamic models. The hysteresis loops in Figure 3(a) can be attributed to the change in stability of the steady-state solution branch either side of two saddle node bifurcations labelled $\mathrm{S}$ in Figure 3(b). The locus of stable steady-state solutions, indicated by the solid black parts of the curve in figure 3(b), are the solutions to which the system converges to over time. Consequently, the dynamic model is able to follow the locus of stable steady-state solutions, provided the parameter variation is sufficiently slow for the system to reach the equilibrium solution. By contrast, the system will tend away from the locus of unstable steady-state solutions, indicated by the dashed part of the curve in figure 3(b). This makes it virtually impossible to follow the unstable solutions in a quasi static simulation, as any slight perturbation from the unstable solution will result in the system trajectory diverging from the unstable equilibrium towards a stable solution. When the applied force $F$ is decreased past the upper limit point, the nearest steady-state solution (for a given force value) is on the lower stable branch. The system therefore jumps from the upper stable branch to the lower stable branch as shown by the left hand arrow in Figure 3(a). When the applied force is increased again, the system will stay on the lower stable branch until the force is increased past the lower-branch limit point. The jump as the force increases past the lower-branch limit point is shown by the right hand arrow in Figure 3(a).

The force values at which the limit points occur in the continuation analysis are quite close to those at which the jumps occur in the dynamic models. This is because the dynamic models approximate the steady-state solutions; in reality transient effects will cause a jump in the dynamic simulation results to occur slightly after a limit point is passed.

\section{Continuation Results for the Overcentre Mechanism Model}
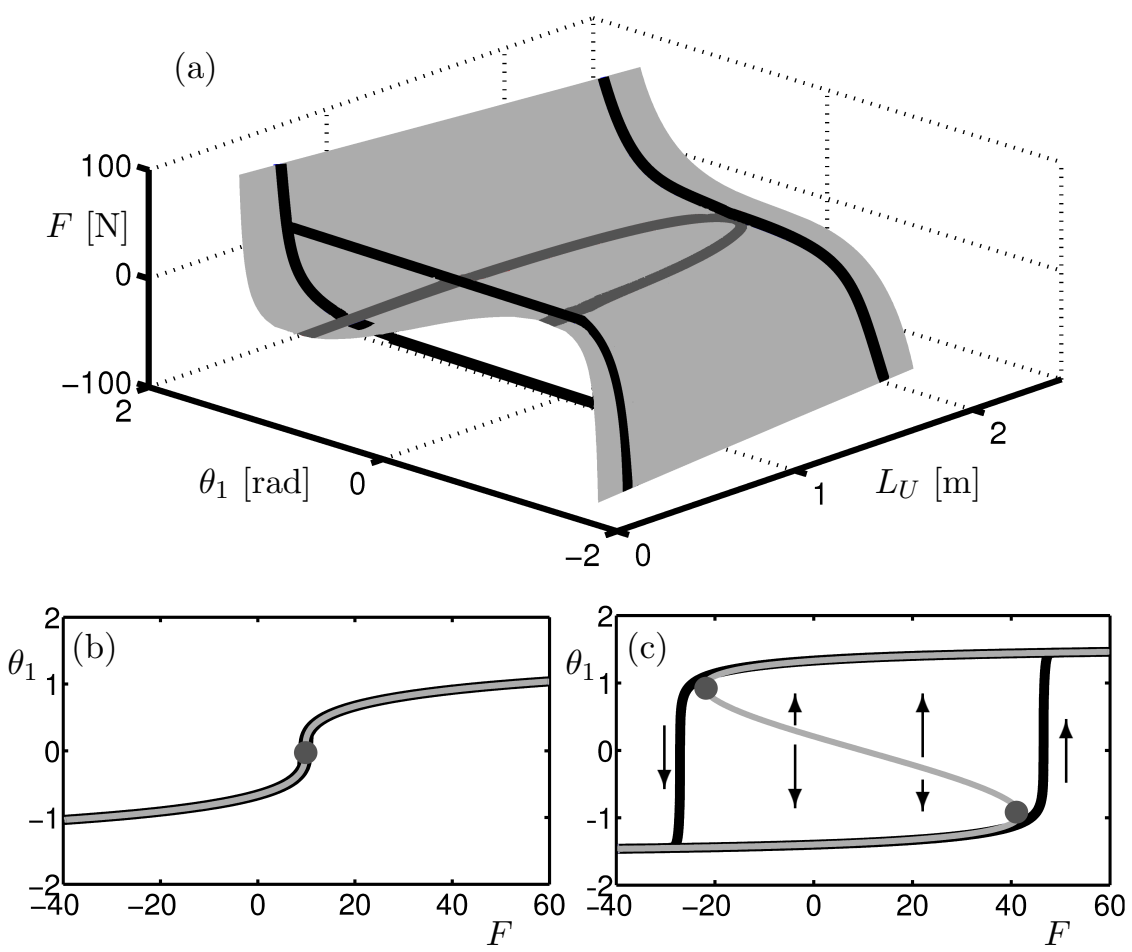

Figure 4. (a) Effect of applied force $F$ and unstrained spring length $L_{U}$ on the steady-state solutions $\theta_{1}$. (b) Effect of applied force on the steady-state solutions with $L_{U}=2 \mathrm{~m}$. (c) Effect of applied force on the steady-state solutions with $L_{U}=0.4 \mathrm{~m}$. The black curves are results from the dynamic model, with continuation results shown by the light grey surface/curves; the limit points are indicated as dark grey curves/points. 
Figure 4 shows a surface of steady-state solutions in terms of the angle $\theta_{1}$, the applied force $F$ and the unstrained spring length $L_{U}$, along with a locus of the limit points. Both were computed by continuation analysis of the static equation formulation. For comparison, multiple quasi-static simulation runs using the dynamic Matlab model were performed for different unstrained spring lengths; two examples are shown as the black curves in Figure 4. The grey surface was created from multiple continuation runs of the static model at discrete unstrained spring lengths, which were interpolated appropriately to produce the surface.

The effect of the limit point can be described by comparing the system response as the applied force is slowly varied. Figure 4(b) shows that, for a given applied force (e.g. 20N), there is a single steady-state equilibrium to which the system will be attracted. If this applied force is slowly decreased, the system will follow the black curve (plotted under the light grey curve) which traces out the locus of equilibria as obtained by the continuation analysis (the grey curve). For all applied force values, there is a unique equilibrium solution which is stable. This is because, as the mechanism is downlocked, the spring remains in compression, so the spring force exerted on the links acts in a constant direction.

Figure 4(c) shows that for a shorter unstrained spring length, two limit point bifurcations appear. These bifurcations indicate that there is a change in stability properties of the equilibrium solutions either side of the bifurcation. For this case, an applied force of $20 \mathrm{~N}$ has two stable equilibrium solutions (the top and bottom curves, shown to be stable because the black, dynamic simulation curve follows these branches) separated by a branch of unstable equilibria. This means that the equilibria to which the system will be attracted in a time history simulation depends on the initial conditions - if the initial system angle (for a given force value) is below the unstable curve, the mechanism will tend towards the lower stable branch, whereas for an initial angle above the unstable branch it will tend towards the top stable branch ${ }^{\mathrm{a}}$. Considering the case when the system starts on the top stable branch as described in the previous section, if the force is decreased the system will follow the black curve which traces the steady-state solutions up to the limit point. If the applied force is increased past the limit point, the system will tend to the single equilibrium which exists on the lower branch, similarly to the case shown in Figure 3. This means that the force at which a limit point bifurcation occurs is the minimum force required to downlock the mechanism.

Even on the level of a two-parameter analysis, the static formulation provides similar results to the dynamic simulations performed in ADAMS. Some of the key advantages of using the static formulation method become apparent when considering the flexibility and ease of computing such surfaces. The ADAMS results are an order of magnitude slower to compute than the continuation results for the same surface. Furthermore, the continuation analysis is able to compute the regions of unstable behaviour bounded by the fold curve without any problem.

\footnotetext{
${ }^{a}$ This type of interpretation requires caution when applied to a multi-DoF system, as a point lying between stable and unstable loci in one projection may not reflect its overall position in full state-space.
} 


\section{Two-parameter Continuation of the Limit Point Locus}

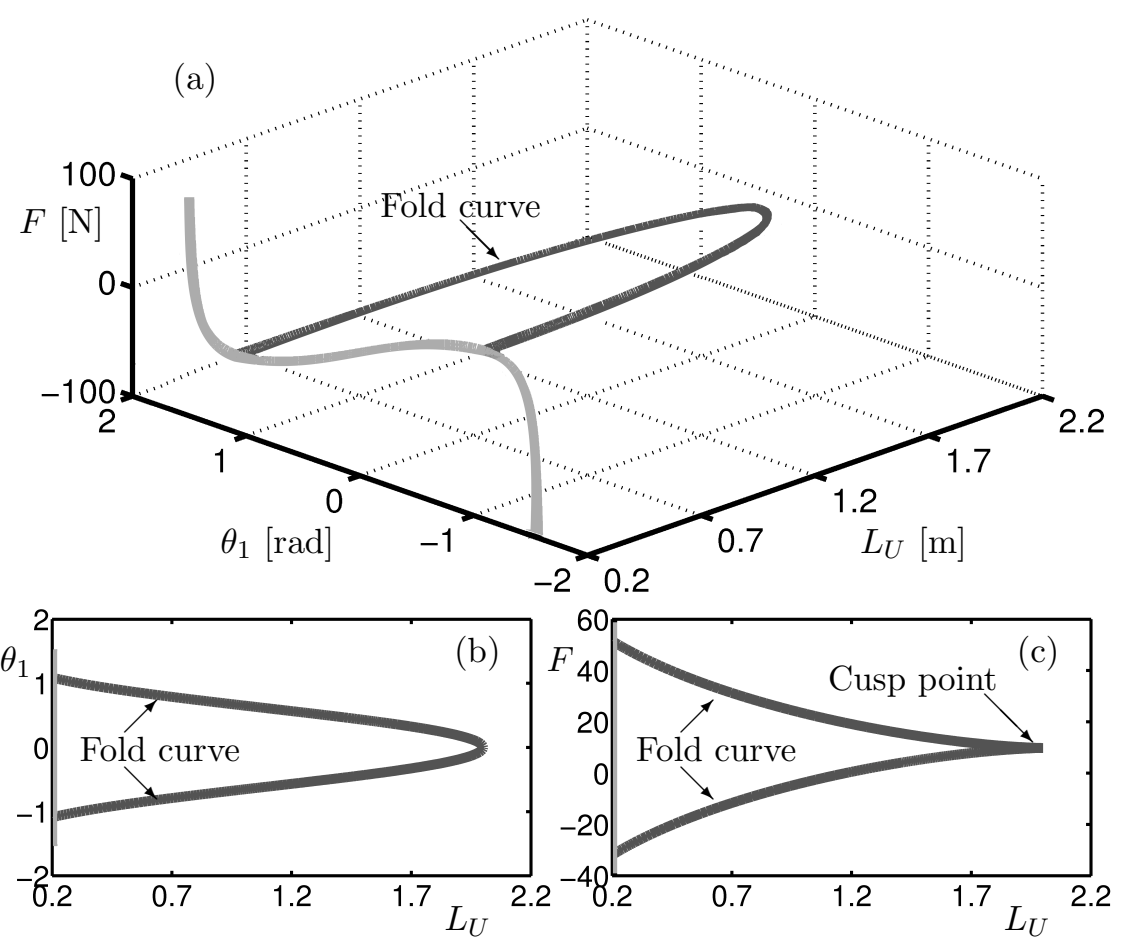

Figure 5. (a) Limit point trace for varying unstrained spring length $L_{U}$, and its projections in the plane of (b) link-1 angle $\theta_{1}$ versus unstrained spring length $L_{U}$ plane and (c) applied force $F$ versus unstrained spring length $L_{U}$.

The flexibility of the static formulation is evident when considering the locus of limit points plotted in Figure 5; this locus is also shown in Figure 4. Importantly, it can be computed directly by using twoparameter continuation without the need to produce a surface of equilibria. Since a bifurcation point has a particular mathematical expression, this expression can be used (effectively) as another system constraint. This allows two parameters to be used as continuation parameters, hence 'two-parameter continuation'. For the overcentre mechanism, the limit point locus was computed by tracing the limit point in terms of the unstrained spring length $L_{U}$ whilst allowing the force $F$ to vary appropriately to maintain equilibrium. Figures 5(b) and (c) show projections of how the two 'dependent' variables, applied force $F$ and the angle of bar one $\theta_{1}$, vary as the unstrained spring length $L_{U}$ is continued. The downlock force value for a given unstrained spring length is found by considering the lower branch of saddle node bifurcations in Figure 5(c). Referring back to Figure 4(c), any initial system configuration with an equilibrium on the top stable branch will remain on this branch unless the applied force is decreased below the upper limit point. In Figure 5(c), the projection shown in Figure 4(c) would appear as a vertical trajectory. Hence, as the force is decreased the overcentre mechanism will remain on the upper branch until the applied force reaches the lower fold curve, when the 'jump' in link angle would occur. From a design perspective, this projection could be used with relative ease to determine upper and lower bounds on the unstrained spring length in dependence on downlock load requirements. For example, if it was desired that the overcentre mechanism must downlock from any position without applying any external forces (i.e. $F \geq 0$ ), Figure 5 (c) shows that this can only be achieved for unstrained spring lengths approximately greater than $1.2 \mathrm{~m}$.

Apart from reducing the computational workload, continuing the limit point ensures comprehensive coverage of the underlying steady-state system behaviour, whereas interpolating between results for discrete spring length values may miss highly nonlinear behaviour. 


\section{Nose Landing Gear Mechanism}

Similarly to the static model of an overcentre mechanism (as described in Section IIB), the equations for the static landing gear model are formulated as a set of algebraic equations with 15 bar position and rotation states (all assumed to be independent). This simplified landing gear mechanism consists of 5 bars, connected to one another by planar revolute joints. The points where the gear attaches to the aircraft are, for the purposes of this model, assumed to be fixed in space. The main fitting attachment point was chosen as the global co-ordinate system origin. The layout of the landing gear, along with various state variables and parameters associated with it, is sketched in Figure 6.

\section{A. Static Model}
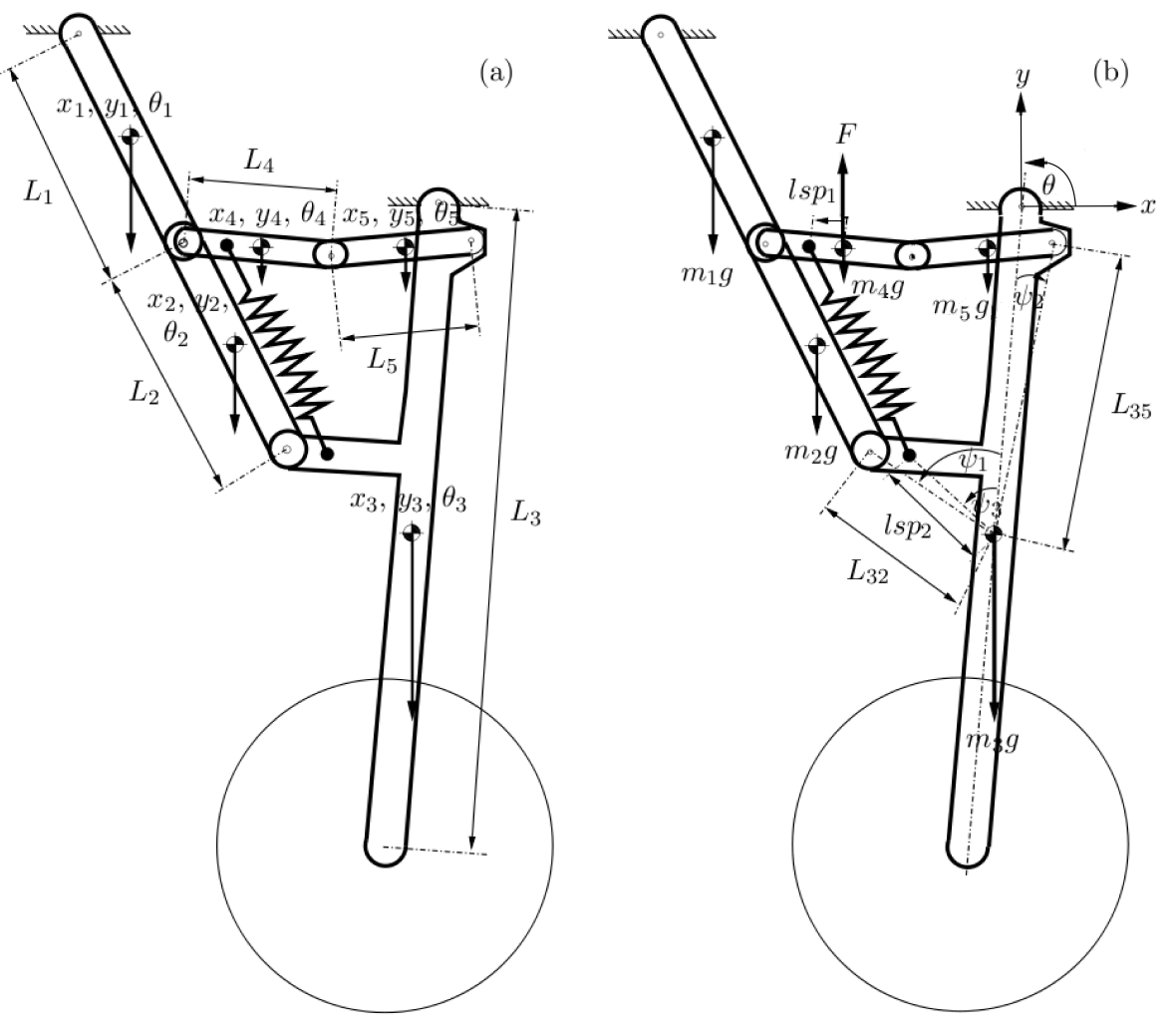

Figure 6. Sketch of a nose landing gear in downlocked position.

Unlike for the overcentre mechanism the force/moment equilibrium conditions are too complicated to express as a single constraint equation. It was therefore necessary to introduce extra states (representing the inter-link forces) to allow the force and moment equilibrium equations for each bar to be expressed as an individual constraint. 
The resulting system of states and equations consists of:

- 10 position $(x, y)$ co-ordinates of the centres of gravity of the 5 links;

- 5 rotation $(\theta)$ co-ordinates of the links' orientations to the positive $x$-axis;

- 14 equations to constrain each bar end to a given point;

- 18 inter-link forces;

- 4 ground-link forces;

- 1 applied actuator force;

- 10 static force equilibrium equations;

- 5 moment equilibrium equations;

- 8 force equilibrium equations at link joints.

There are therefore 37 equations described in terms of 37 states and several parameters. When performing a continuation run, AUTO requires 37 states and one additional continuation parameter to be determined by 37 equations. By using static equilibrium equations it is possible to use any model state or parameter as the continuation parameter, provided there are still 37 variables to be determined.

To reduce the complexity, it is possible to remove eight inter-link forces by applying the eight force equilibrium equations at link joints directly. The description of the four ground-link forces requires four equations, so a further four equations and states can be removed. These two simplifications reduce the system by 12 equations and 12 forces, resulting in a system of 25 equations with 25 states and several additional parameters. The geometric constraints are:

$$
\begin{aligned}
& x_{1}-A_{x}-\frac{L_{1}}{2} \cos \theta_{1}=0 \\
& y_{1}-A_{y}-\frac{L_{1}}{2} \sin \theta_{1}=0 \\
& x_{1}-x_{2}+\frac{L_{1}}{2} \cos \theta_{1}+\frac{L_{2}}{2} \cos \theta_{2}=0 \\
& y_{1}-y_{2}+\frac{L_{1}}{2} \sin \theta_{1}+\frac{L_{2}}{2} \sin \theta_{2}=0 \\
& x_{2}-x_{3}+\frac{L_{2}}{2} \cos \theta_{2}-\frac{L_{23}}{2} \cos \left(\theta_{3}+\psi_{1}\right)=0 \\
& y_{2}-y_{3}+\frac{L_{2}}{2} \sin \theta_{2}-\frac{L_{23}}{2} \sin \left(\theta_{3}+\psi_{1}\right)=0 \\
& x_{3}+\frac{L_{3}}{2} \cos \theta_{3}=0 \\
& y_{3}+\frac{L_{3}}{2} \sin \theta_{3}=0 \\
& x_{1}-x_{4}+\frac{L_{1}}{2} \cos \theta_{1}+\frac{l l_{1}}{2} \cos \theta_{4}=0 \\
& y_{1}-y_{4}+\frac{L_{1}}{2} \sin \theta_{1}+\frac{l l_{1}}{2} \sin \theta_{4}=0 \\
& x_{4}-x_{5}+\frac{l l_{1}}{2} \cos \theta_{4}+\frac{l l_{2}}{2} \cos \theta_{5}=0 \\
& y_{4}-y_{5}+\frac{l l_{1}}{2} \sin \theta_{4}+\frac{l l_{2}}{2} \sin \theta_{5}=0 \\
& \left.\begin{array}{r}
x_{5}-x_{3}+\frac{l l_{2}}{2} \cos \theta_{5}-\frac{L_{35}}{2} \cos \left(\theta_{3}-\psi_{2}\right)=0 \\
y_{5}-y_{3}+\frac{l l_{2}}{2} \sin \theta_{5}-\frac{L_{35}}{2} \sin \left(\theta_{3}-\psi_{2}\right)=0
\end{array}\right)
\end{aligned}
$$

The various elements within the force/moment equilibrium equations can be expressed in the matrix form $A \bar{F}-B=0$, where $\bar{F}$ is a vector of the inter-link forces, $A$ is a matrix of force coefficients and $B$ is a vector of the remaining terms. We have: 


$$
A=\left[\begin{array}{ccccccccccc}
s \theta_{1} & -c \theta_{1} & 0 & 0 & 0 & 0 & s \theta_{1} & -c \theta_{1} & 0 & 0 & 0 \\
1 & 0 & -1 & 0 & 0 & 0 & 0 & 0 & 0 & 0 & 0 \\
0 & 1 & 0 & -1 & 0 & 0 & 0 & 0 & 0 & 0 & 0 \\
0 & 0 & s \theta_{2} & -c \theta_{2} & 0 & 0 & 0 & 0 & 0 & 0 & 0 \\
0 & 0 & {[C](1)} & {[C](2)} & {[C](3)} & {[C](4)} & 0 & 0 & 0 & 0 & 0 \\
0 & 0 & 0 & 0 & 0 & 0 & 1 & 0 & 1 & 0 & 0 \\
0 & 0 & 0 & 0 & 0 & 0 & 0 & 1 & 0 & 1 & 1 \\
0 & 0 & 0 & 0 & 0 & 0 & 0 & 0 & s \theta_{4} & -c \theta_{4} & -\frac{1}{2} c \theta_{4} \\
0 & 0 & 0 & 0 & -1 & 0 & 0 & 0 & 1 & 0 & 0 \\
0 & 0 & 0 & 0 & 0 & -1 & 0 & 0 & 0 & 1 & 0 \\
0 & 0 & 0 & 0 & s \theta_{5} & -c \theta_{5} & 0 & 0 & 0 & 0 & 0
\end{array}\right]
$$

where $s \equiv \sin , c \equiv \cos$ and

$$
\begin{aligned}
{[C]=\left[\begin{array}{c}
L_{32} \sin \left(\theta_{3}+\psi_{1}\right)-\frac{L_{3}}{2} \sin \theta_{3} \\
\frac{L_{3}}{2} \cos \theta_{3}-L_{32} \cos \left(\theta_{3}+\psi_{1}\right) \\
L_{35} \sin \left(\theta_{3}-\psi_{1}\right)-\frac{L_{3}}{2} \sin \theta_{3} \\
\frac{L_{3}}{2} \cos \theta_{3}-L_{35} \cos \left(\theta_{3}-\psi_{1}\right)
\end{array}\right]^{T}, } \\
B=\left[\begin{array}{l}
\frac{m_{1}}{2} g \cos \theta_{1} \\
0 \\
m_{2} g \\
\frac{m_{2}}{2} g \cos \theta_{2} \\
-\frac{m_{3}}{2} g L_{3} \cos \theta_{3}-\left[l_{s p 2} \sin \left(\theta_{3}+\psi_{3}\right)-\frac{L_{3}}{2} \sin \theta_{3}\right] F_{s 3}^{x}+\left[l_{s p 2} \cos \left(\theta_{3}+\psi_{3}\right)-\frac{L_{3}}{2} \cos \theta_{3}\right] F_{s 3}^{y}+D \\
-F_{s 4}^{x} \\
m_{4} g-F_{s 4}^{y} \\
\frac{1}{2} F_{s 4}^{y}\left(1-\frac{l_{s p 1}}{L_{4}}\right) \cos \theta_{4}-\frac{1}{2} F_{s 4}^{x}\left(1-\frac{l_{s p 1}}{L_{4}}\right) \sin \theta_{4}-\frac{m_{4}}{2} g \cos \theta_{4} \\
0 \\
-m_{5} g \\
-\frac{m_{5}}{2} g \cos \theta_{5}
\end{array}\right],
\end{aligned}
$$

with

$$
D=\frac{1}{4} \rho\left(U_{\infty} \cos \left(\alpha-\theta_{3}+\frac{\pi}{2}\right)^{2} d\left(L_{3}^{2}\right) C_{D}\right.
$$

Here:

$\rho$ is the air density at sea level $=1.225 \frac{\mathrm{kg}}{\mathrm{m}^{3}}$

$U_{\infty}$ is the air velocity;

$\alpha$ is the angle between the airflow direction and the global x-axis, initially set to zero;

$d$ is the diameter of the main fitting;

$C_{D}$ is an estimate of the drag coefficient of the main fitting of the landing gear $=1.17^{\mathrm{b}}$.

By expressing the force equilibrium equations in this way, initialising the solution when matrix $A$ and vector $B$ are known only requires computing $\bar{F}=A^{-1} B$.

\section{B. Downlock Force Analysis}

This section demonstrates how the continuation approach can be used to determine the downlock force required to engage the lock links for a planar nose landing gear mechanism under different aerodynamic loads and spring configurations. Subsection 1 presents a single parameter continuation investigation, which is built upon in Subsection 2, where results from a two-parameter continuation analysis are presented and discussed.

\footnotetext{
bassuming the shock strut is the main contributor to drag and that its drag can be approximated by that of a cylinder.
} 


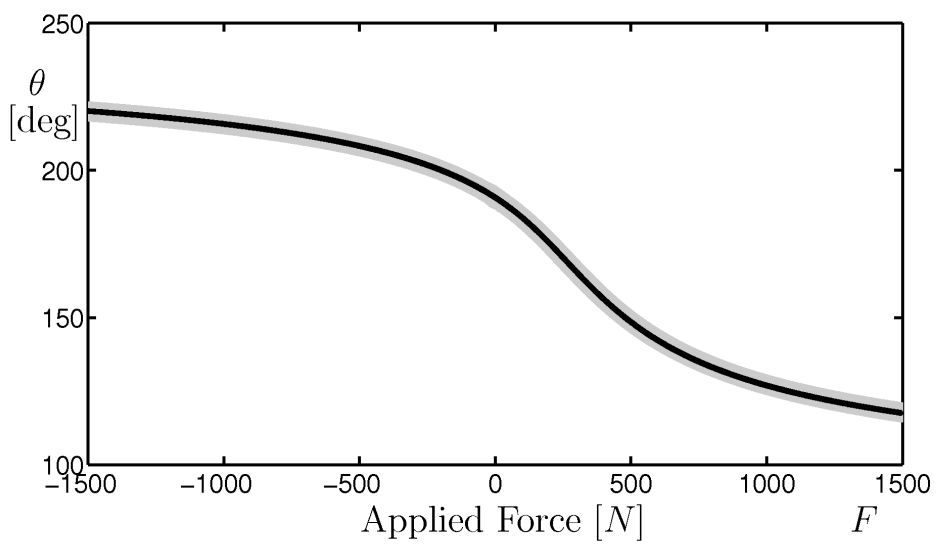

Figure 7. Overcentre angle $\theta$ versus applied force $F$ for a dynamic model (light grey curve) and the staticalgebraic continuation model (black curve).

The continuation algorithm in AUTO was used to solve for equilibria as the applied force $F$ (see Figure 6 ) varies - initially with no aerodynamic or spring loading. The resulting variation in the overcentre angle $\theta$ (the angle between the two locklinks) is shown in Figure 7. As with the overcentre mechanism, an equivalent dynamic model was created to act as a baseline for validation purposes. The light grey curve in Figure 7 was the response obtained from the simple dynamic model created in ADAMS. It can be seen that the static-algebraic continuation model produces an identical response (black curve, Figure 7), suggesting that the formulation is correct.

It was reasoned that for the gear to downlock the lock links must pass through the horizontal plane, so the downlock force was taken to be the value of force $F$ when the rotation angle $\theta_{4}=0^{\mathrm{c}}$. The following results were obtained by mathematically constraining the lock links to be horizontal. This was achieved by using $\theta_{4}$ as a fixed parameter, whilst the applied force $F$ was allowed to vary as a state. By making $F$ a state, static equilibrium can be maintained as a different parameter (e.g. spring stiffness) is varied as the continuation parameter. It should be noted that, although the force $F$ is referred to as the downlock load herein, for a planar gear this force is more akin to an 'unlock force', because its positive direction of action signifies that the gear will downlock without the need to be forced. An unlock actuator, however, would need to work against the structural weight, aerodynamic loads and downlock springs to unlock the deployed gear.

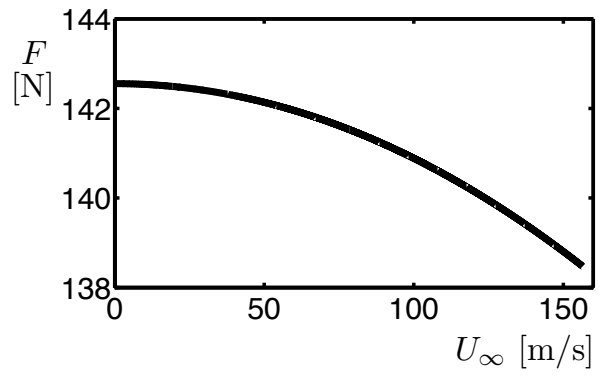

Figure 8. Effect of airflow $U_{\infty}$ on downlock force $F$.

With the addition of aerodynamic loads, Figure 8 shows the variation in downlock force as a function of aircraft velocity. The effect of airspeed on the downlock force is minimal; an increase in airspeed from 0

${ }^{\mathrm{c}}$ equivalent to an overcentre angle $\theta=180^{\circ}$ because the two link attachment points are exactly level when downlocked; see Figure 6 . 
to $100 \mathrm{~m} / \mathrm{s}$ causes a decrease in the downlock load of only $1.5 \mathrm{~N}$. The quadratic relationship between the airflow and downlock force is due to the $U_{\infty}^{2}$ term in the drag force approximation, and suggests that the downlock load is directly proportional to the drag force.
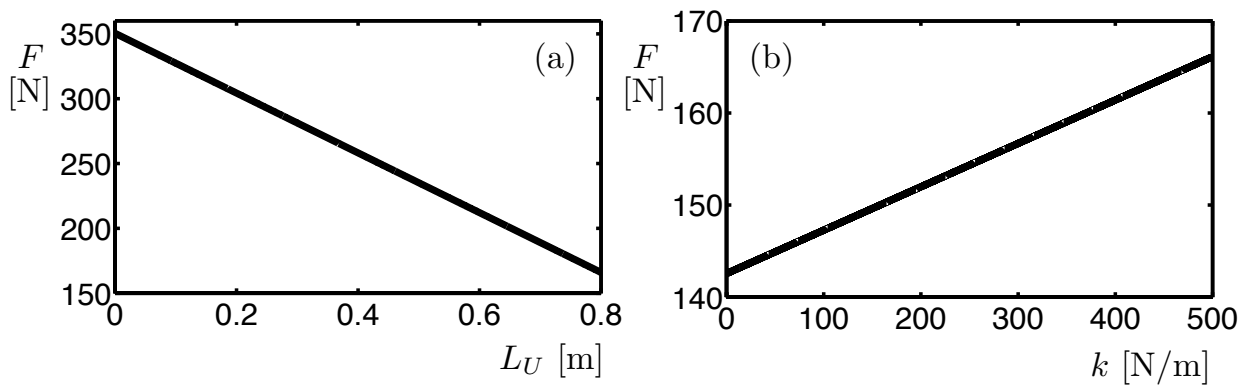

Figure 9. Spring property effects on downlock loads, showing: effect of (a) unstrained spring length $L_{U}$ and (b) of spring stiffness $k$ on the downlock force $F$.

The effect of increasing spring stiffness and unstrained spring length is shown in Figure 9. As for the overcentre mechanism, the spring stiffness has a linear effect on the downlock force. Unlike for the overcentre mechanism, the downlock load is also linearly dependent on unstrained spring length. The landing gear's spring is always in tension and acts in approximately the same direction as the applied force. By contrast, in the overcentre mechanism the spring experiences tension and compression whilst acting perpendicularly to the applied force; it is this perpendicular action of the spring force to the applied force which creates the hysteresis loop described previously.

In order to replicate the results shown in Figures 8 and 9 using the traditional approach, the applied force would first need to be varied slowly to engage the lock links. This would provide an equivalent result to the initial continuation run shown in Figure 7. A parameter would then need to be changed and the simulation repeated. The traditional approach would therefore require a series of runs at small enough parameter intervals to generate a sufficiently smooth curve of solutions: there is the potential for dynamic simulations to miss areas of interest if too few simulations are performed.

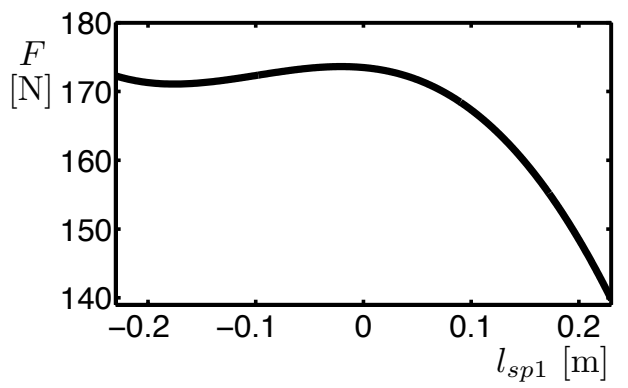

Figure 10. Effect of spring attachment point position on downlock force.

In spite of the linear influence of spring stiffness and unstrained spring length, Figure 10 shows that the relationship between the applied force and the position of the lock-link-spring attachment point is nonlinear. This is because moving the lock-link-spring attachment point from the end nearest the sidestays $\left(l_{s p 1}>0\right)$ to the end where the two lock links join $\left(l_{s p 1}<0\right)$ affects the applied force in several ways:

- the moment created by the spring force about the lock-link-sidestay joint increases, so the applied force must also increase in order to maintain static equilibrium;

- the distance between the spring ends (and hence the spring length) decreases, so the decreasing spring force allows the applied force to also decrease;

- internal forces will change as the force in the spring (and hence the force applied at both ends of the spring) changes. 
It is a combination of these three nonlinear effects that leads to the relationship shown in Figure 10.

\section{Two-parameter Continuation of the Limit Point Locus}

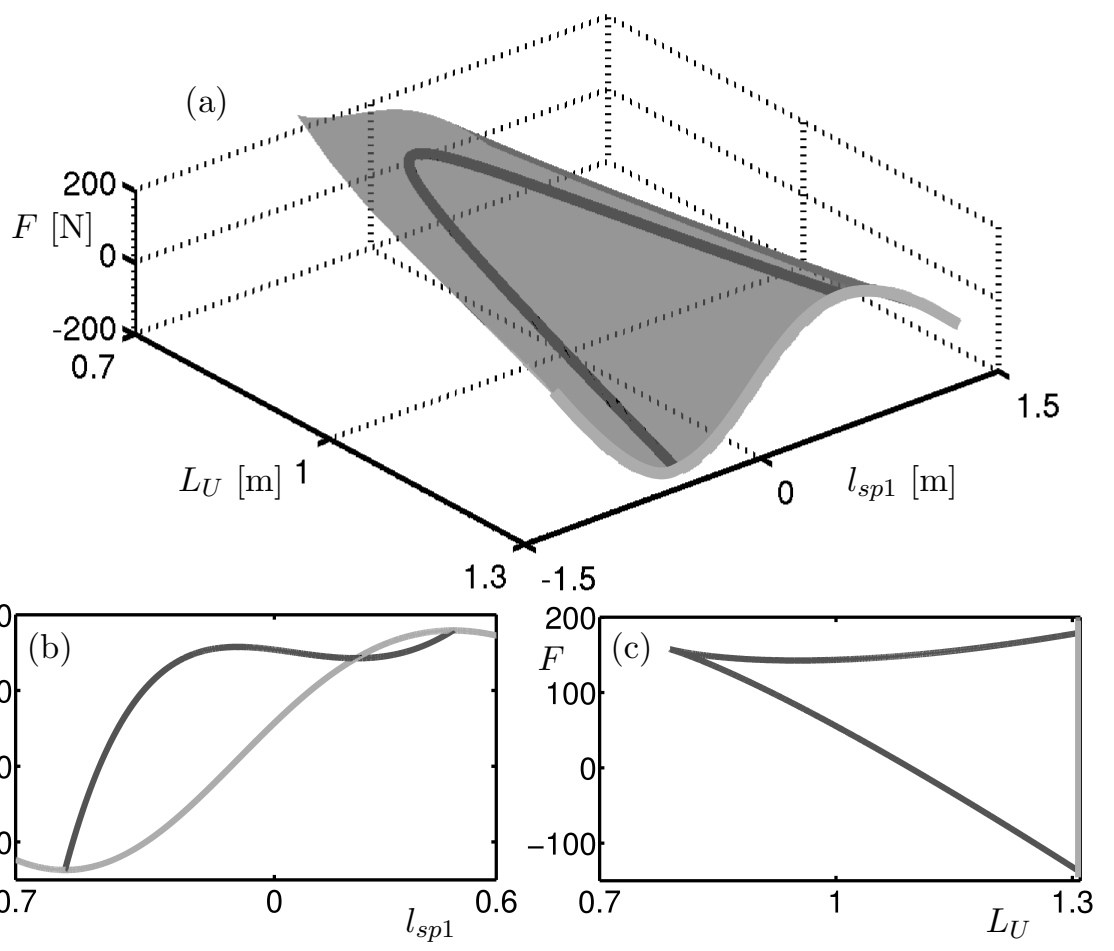

Figure 11. Limit point variation under two-parameter continuation of a nose landing gear mechanism with projections (a) on the space of spring attachment point $l_{s p 1}$, unstrained spring length $L_{U}$ and applied force $F$, and on planes of (b) applied force $F$ versus spring attachment point $l_{s p 1}$ and (c) applied force $F$ versus unstrained spring length $L_{U}$. The surface of equilibria is in light grey with the locus for $L_{U}=1.3 \mathrm{~m}$ highlighted on the surface and shown in the two projections. The limit point locus is in dark grey.

Figure 11 shows the locus of the fold points as a function of unstrained spring length $L_{U}$ and lock-linkspring attachment point $l_{s p 1}$, along with the corresponding surface of equilibria. Figure 11(c) shows that the limit points are only present for unstrained spring lengths of greater than $0.8 \mathrm{~m}$. As with the overcentre mechanism, the limit points come together and disappear at a cusp point (Figure 11(c)). Unlike for the overcentre mechanism, the physical interpretation of the limit points is less intuitive because, in reality, the spring attachment point position is a fixed parameter rather than a state. The physical interpretation requires consideration of these results from a design perspective, as it is during the design phase that geometric parameters can actually be varied to meet an objective.

From a design perspective, it could be important to know how changing the spring attachment point would affect the downlock load. For the majority of unstrained spring lengths, decreasing $l_{s p 1}$ causes the downlock load to increase. By definition, the gradient of the equilibrium locus changes either side of the local maximum/minimum equilibrium points in the $\left(F, l_{s p 1}\right)$-plane; see the light grey curve in Figure 11(a). The limit points traced in Figure 11 (dark grey curve) bound the region in which moving the spring attachment point from the sidestay end to the lock-link-lock-link joint end (i.e. decreasing $l_{s p 1}$ ) causes the applied force to decrease - the opposite result to that expected intuitively.

It would be impractical to reproduce these results using dynamic simulations, because a separate time history run would be needed for every point on the surface in Figure 11. 


\section{Conclusion}

It has been shown that continuation methods, when applied to mechanisms expressed as a set of static equilibrium equations, provide steady-state solutions that are as accurate as traditional dynamic models. Solving static models in parameter space with a continuation algorithm offers significant reductions in computational times when compared to more traditional, dynamic simulation based approaches. This is because simpler models can be used and points of interest, such as the limit points, can be traced out directly under multiple parameter variations. The results from the overcentre mechanism example show that it is also possible to follow unstable equilibria (determining boundaries of bistability) with continuation methods, something which cannot easily be achieved by dynamic simulations. The feasibility of this continuation approach when applied to a complex mechanism became apparent from the nose landing gear mechanism investigation. The results for the nose landing gear model also demonstrate the flexibility of the analysis offered by expressing the landing gear as a set of static equilibrium equations. It was shown that system dependencies can be determined readily upon variation of any state or system parameter. This is a definite advantage over conventional dynamic simulations. For both the overcentre and nose landing gear mechanisms, examples were presented of how design criteria could potentially be derived from the continuation analysis. The computational efficiency and flexibility achieved with the continuation approach makes it highly suitable for analysing more complex three-dimensional mechanisms, such as a dual sidestay landing gear mechanism. For a dual sidestay landing gear, the mechanism can be described in terms of 36 geometric states and 20 internal force states if using the static equation method. Besides the increase in model states, the nature of a dual sidestay landing gear mechanism is different from the nose landing gear mechanism investigated here because the dual sidestay is over-constrained, resulting in a highly sensitive downlock solution which only exists if all the downlock constraints are exactly satisfied. The continuation approach described here would be well suited to investigating this highly nonlinear system in an efficient and thorough manner.

\section{Acknowledgments}

This research was supported by an Engineering and Physical Sciences Research Council (EPSRC) Case Award grant in collaboration with Airbus in the UK.

\section{References}

\footnotetext{
${ }^{1}$ Lyle, K.H., Jackson, K.E., Fasanella, E.L., Simulation of Aircraft Landing Gears with a Nonlinear Dynamic Finite Element Code, AIAA Journal of Aircraft, Vol. 39, No. 1, January - February 2002.

${ }^{2}$ Kruger, W., Besselink, I., Cowling, D., Doan, D.B., Kortum, W., Krabacher, W., Aircraft Landing Gear Dynamics: Simulation and Control, Vehicle System Dynamics, Vol. 28, 1997.

${ }^{3}$ Conway, H.G., Landing Gear Design. Chapman and Hall, London, 1958.

${ }^{4}$ Currey, N.S., Aircraft Landing Gear Design: Principles and Practices. AIAA, Washington D.C, 1988.

${ }^{5}$ Roskam, J., Airplane Design. Part 4, Layout design of landing gear and systems, Roskam Aviation and Engineering Corporation, Ottawa, 1986.

${ }^{6}$ Strogatz, S., Nonlinear dynamics and chaos, Springer, 2000.

${ }^{7}$ Guckenheimer, J. and Holmes, P., Nonlinear Oscillations, Dynamical Systems and Bifurcations of Vector Fields, Applied Mathematical Sciences Vol. 42, Westview Press, February 2002.

${ }^{8}$ Krauskopf, B., Osinga, H. M., and Galàn-Vioque, J., Numerical Continuation Methods for Dynamical Systems, Springer, 2007.

${ }^{9}$ Rankin, J., Coetzee, E., Krauskopf, B. and Lowenberg, M., Bifurcation and Stability Analysis of Aircraft Turning on the Ground, AIAA Journal of Guidance, Dynamics and Control, Vol. 32, No. 2, March 2009.

${ }^{10}$ Thota, P., Krauskopf, B. and Lowenberg, M., Interaction of Torsion and Lateral Bending in Aircraft Nose Landing Gear Shimmy, Nonlinear Dynamics, 57(3), 2009.

${ }^{11}$ Doedel, E., Champneys, A., Fairgrieve, T., Kuznetsov, Y., Sandstede, B., and Wang, X., AUTO 97 : Continuation and bifurcation software for ordinary differential equations, http://indy.cs.concordia.ca/auto/, May 2001.

${ }^{12}$ Udwadia, F.E. and Kalaba, R.E., Analytical Dynamics: A New Approach. Cambridge University Press, New York, 1996.
} 